\title{
ANALYZING THE EFFICIENCIES OF HOSPITALS: AN APPLICATION OF DATA ENVELOPMENT ANALYSIS
}

\author{
M. Sahin Gok, \\ Bulent Sezen, \\ Gebze Institute of Technology, Turkey
}

\begin{abstract}
The aim of this study is to investigate the efficiencies of hospitals in Turkey with respect to their ownerships (i.e. state, education \& research, university and private) for the years 2001 to 2006 . The impact of health care reforms on the efficiencies is also examined in order to highlight possible policy implications for policy makers. Comparative performance evaluation of hospitals has been achieved by using Data Envelopment Analysis (DEA) as well as Malmquist Index calculations (i.e. to determine the direction and degree of yearly changes in performance), super efficiency analysis and slack evaluations. According to the findings, hospital ownership significantly influences hospital efficiencies. The influence of health care reforms on hospital efficiency has been observed especially for the state and private hospitals. The average efficiencies of state hospitals remarkably increase while the average efficiencies of private hospitals decrease especially after the starting of reforms in the stateowned hospitals. This paper adds value to the current body of research by addressing the impact of hospital ownerships and health care reforms to the efficiencies of Turkish hospitals. There are similar studies of Data Envelopment Analysis applied for hospitals in several other countries. Researchers may compare the results of this study with the findings of other studies.
\end{abstract}

Keywords Hospital efficiency, Hospital ownership, Data Envelopment Analysis, Turkey

\section{INTRODUCTION}

In today's dynamic and rapidly changing socio-economic conditions, all institutions have to search and find ways for continuous improvement. As a service business, health care institutions have followed the similar goals with other organizations for achieving performance improvements. In recent years, efficiency has been one of the most important issues for hospitals which used limited resources for maximum value (Chu et al., 2003).

Efficiency is defined as the ratio of outputs to the resources used. One way to increase efficiency is to decrease the level of resources and investments and/or increase the production factors. However, health care is a sector in which human factor is the most critical issue. Social and political concerns force the authorities to make new investments for providing better health services especially in developing countries such as Turkey. In fact, financial and non-financial investments have increased since late $90 \mathrm{~s}$ in the public and private hospitals in Turkey. Medical technologies, number of beds, doctors and medical staff have been improved. Also teaching hospitals have benefited from these improvement efforts with increased supports for scientific researches and projects. To better understand the outcomes of these investments, changes in efficiency levels of the Turkish hospitals had to be investigated.

One of the major considerations of hospital performance evaluation is the impact of ownership on the efficiency. Turkish hospitals are arranged in four groups by the ownership types: (i) public hospitals, (ii) education and research hospitals, (iii) university hospitals and (iv) private hospitals. There are many previous studies investigated the influence of ownership on hospital efficiency in several countries (e.g., White and Ozcan, 1996; Grosskopf et al., 2001; Burgess and Wilson, 1998; Hofmarcher et al., 2002; Helmig and Lapsley, 2001; Ramanathan, 2005; Gannon, 2005).

Data Envelopment Analysis (DEA) is a widely used technique for the efficiency measurement of hospitals. It is popular in evaluating hospital efficiency because it is applicable to the multiple inputoutput that is essential for the nature of a health care system (Hollingsworth et al., 1999). In this study, number of specialists, number of medical practitioners and number of beds are used as input variables; while number of outpatients, discharge number, number of surgical operations (categorized as small, medium and large), number of births, bed utilization rate, average inpatient days, bed turnover rate, and ratio of inpatients to outpatients are used as output variables. 


\section{ASSESSING THE EFFICIENCIES OF HOSPITALS}

In recent years efficiency has become one of the most attractive work areas of healthcare management literature. Some authors argue that hospitals are profit organizations while some others do not agree with them (White and Ozcan, 1996). Hospitals, whether are economic organizations or not (Ferrier and Valdmanis, 2004), have limited resources to gain maximum value like all other organizations (Watcharasriroj and Tang, 2004; Harris et al., 2000).

Studies on hospital efficiency mostly focus on the issue of maximum gain with limited resources (Sorkis and Talloru, 2002). One of the frequently raised issues on these studies is the efficient use of resources and controlling the costs. Thus, the interest on hospital efficiency has increased because of the desire to control the increasing costs. Accordingly, hospital resources and their processes became critical and, as a result, the number of studies has increased in recent years.

Regression analysis, ratio analysis and non-parametric techniques were applied to analyze the hospital efficiency in the previous studies (Ferrier and Valdmanis, 2004). DEA is one of the most applied technique for evaluating hospital efficiency (Linna et al., 2006; Bakar et al., 2010). DEA enables the use of multiple inputs and outputs at the same time for hospital efficiency studies.

Literature review of DEA studies on hospital efficiency shows that there are a number of studies applied in USA, Austria, Jordan, Germany, Greece, Oman, Taiwan, Spain, Thailand, Africa (Botswana), Norway, Ireland, and Finland. Number of beds, specialists, medical practitioners, medical stuff, and manager are seen to be most frequently used input variables in these studies. Number of inpatients, outpatients, surgical operations, visitors, and patient days are seen to be most frequently used output variables. Also degree of training, technology, number of clinic, laboratory, morbidity, mortality, and proprietary capital, costs of medical services, management, discharge, payment, and total profit are seen to be used for DEA variables in such studies.

Several researches in the past focused on the effect of hospital ownership on hospital performance (e.g. Biqrn et al., 2003; Burgess and Wilson, 1998; Chang 1998; Chang et al., 2004; Gannon, 2005; Grosskopf et al., 2001; Helmig and Lapsley, 2001; Hofmarcher et al., 2002; Ramanathan, 2005; White and Ozcan, 1996). Most of these studies found that hospital ownership has a critical role on performance. In addition, Biqrn et al., (2003), Grosskopf et al., (2001), Helmig and Lapsley (2001), and Ramanathan (2005) used Malmquist Productivity Index (MPI) to evaluate the performance changes in time series. In line with the past studies, the influence of hospital ownership on the efficiency of Turkish hospitals and the efficiency changes in years 2001 and 2006 have been analyzed.

\section{DATA ENVELOPMENT ANALYSIS}

DEA has become a widely used technique for efficiency measurement. It is mainly based on the earlier concept of Frontier Analysis (Farrel, 1957) and it became popular after it was first introduced in the article by Charnes et al. (1978). It assesses the relative efficiency scores of a particular set of Decision-Making-Units (DMU), which produce a variety of outputs by using several inputs. This approach uses a mathematical programming method to create a set of weights for each inputs and outputs, which considers how efficiency in the DMUs can be improved, and ranks individual DMUs based on efficiency score (Liu et al., 2007; Bakar et al., 2010). DEA computes each hospital's efficiency by maximizing the ratio of the weighted sum of output variables and the weighted sum of input variables (Kumar, 2010). DEA has been used for measuring the efficiency of many other application areas including banks, airlines, universities, etc.

DEA evaluates the relative technical efficiency with linear programming model by using input and output variables from similar and homogeneous decision making units (DMU). DEA has two key advantages for efficiency analysis: (1) it readily analyzes multiple inputs and outputs at the same time therefore, (2) captures more specific production characteristics of each unit (Ferrari, 2006). DEA model calculates an efficiency score for each DMU. Efficiency scores of DMUs which are equal to 1 are called "efficient" and different to 1 are called "inefficient" (Zhu, 2003). DMUs' scores are compared with one another and the set of most effective DMUs is called "efficiency frontier" (Junoy, 2000). Consequently, as Kumar and Gulati (2008) stated, DEA outlines an efficiency frontier over the data points to determine the efficiency of each DMU relative to this frontier. In this benchmark model there are two assumptions: (1) input oriented (while outputs are hold constant and inputs are decreased), (2) output oriented (while inputs are hold constant and outputs are increased), (Harris et al., 2000). Although some authors used output oriented approach to analyze hospital performance with 
DEA (e.g., Biqrn et al., 2003; Hu and Huang, 2004) the majority of the past studies suggested the input oriented approach for measuring hospital efficiency (e.g., Ferrier and Valdmanis, 2004; Lynch and Ozcan, 1994; O'neill and Dexter, 2004). The reason is that the selected input variables in most of the studies are more controllable than the outputs.

The first DEA model developed by Charnes et al. (1978), named the CCR model, was based on the assumption of Constant Return to Scale (CRS). Later, Banker et al. (1984) enhanced the CCR model and developed the BCC model using the Variable Return to Scale (VRS), in 1984. Consequently, Nayar and Ozcan (2008) concluded that DEA is constructive technique for health care managers to investigate opportunities in accordance to efficiency improvement. Kirigia et al. (2008) state that in the context of hospitals, efficiency means providing maximum services out of obtainable resources or minimizing the use of available resources to produce a given level of services.

\section{Mathematical Structure of DEA}

DEA aims to find decision making units (DMUs) that produce the highest levels of outputs by using the lowest levels of inputs. Therefore, it maximizes the ratio of weighted outputs to weighted inputs for the DMU under consideration. And this maximization objective is subject to the constraint that the same ratio for all DMUs be less than or equal to one. This leads to the following model, in which one can find the efficiency value for $\mathrm{DMU}_{\mathrm{m}}$ (Ramanathan, 2005):

$$
\operatorname{Max} \frac{\sum_{j=1}^{J} v_{m j} y_{m j}}{\sum_{i=1}^{I} u_{m i} x_{m i}}
$$

$$
\begin{gathered}
0 \leq \frac{\sum_{j=1}^{J} v_{m j} y_{n j}}{\sum_{i=1}^{I} u_{m i} x_{n i}} \leq 1 ; n=1,2, \ldots \ldots ., N \\
v_{m j}, u_{m i} \geq 0 ; i=1,2, \ldots \ldots, I ; j=1,2, \ldots . ., J
\end{gathered}
$$

Here, $i$ is the index for inputs, $j$ is the index for outputs, and $n$ is the index for DMUs. The variables $\mathrm{v}_{\mathrm{mj}}$ and $\mathrm{u}_{\mathrm{mi}}$ are the weights representing the importance of each input and output. If the efficiency is equal to 1 , the $\mathrm{DMU}_{\mathrm{m}}$ is located on the efficiency frontier. Here, the efficiency value is a relative measure indicating how $\mathrm{DMU}_{\mathrm{m}}$ operates compared to the other DMUs that are included in the sample.

\section{INPUTS AND OUTPUTS}

Since DEA can evaluate multiple inputs and outputs at the same time, it is accepted as being one of the most effective methods for relative efficiency evaluations. However, selection of inputs and outputs is the most important stage of the analysis process (Nyhan and Peter, 2000). Especially in service businesses, like hospitals, it becomes even more complex to accurately identify inputs and outputs. Thus, to come up with reliable results by using DEA begins with the selection of appropriate inputs and outputs that can be aggregated into a composite index of overall performance standards (Min et al., 2009). The literature review and several interviews with hospital managers revealed the following input variables: number of specialists (x1), number of medical practitioners (x2) and number of beds (x3).

Burgress and Wilson (1998) identified the intellectual capital as being one of the essential inputs of service institutions like hospitals. They also emphasize that doctors are one of the most important arguments for intellectual capital. To illustrate the experience and education levels of doctors, they have been classified in two groups: specialists and medical practitioners (Athanassopoulos et al., 1999).

After defining the input variables, critical output variables should be defined. For this reason, firstly, the products of hospitals should be analyzed. As Burgress and Wilson (1998) noted, the products of hospitals are composed of patient and patient related issues. Accordingly, Harris et al. (2000) emphasized the importance of the number of patients in the efficiency evaluation process. Outputs, in this study, are composed of ten variables. In order to analyze the number of patients, two output variables are defined: ( $\mathrm{y} 1)$ number of outpatients and $(\mathrm{y} 2)$ number of discharge. In addition to these, number of surgical operations (categorized as small (y3), medium (y4) and large (y5)), number of births (y6), bed utilization rate (y7), average inpatient days (y8), bed turnover rate (y9), and ratio of inpatients to outpatients (y10) are also used as other output variables. 
The number of surgeries is used in many studies as a critical factor for evaluating the efficiency of hospitals (e.g., Grosskopf et al., 2001; Burgess and Wilson, 1998; Sorkis and Talloru, 2002). The number of surgeries is grouped as large (y3), medium (y4) and small (y5) surgeries. By this way, medical operations are also included as a producing factor of hospitals. As Grosskopf et al. (2001) states surgery times could better illustrate the functional efforts of hospitals towards their patients. Grouping the number of surgeries as large, medium and small enables the evaluation of the way and size of service provided to the patients (Athanassopoulos et al., 1999).

\section{Data}

To analyze the effects of hospital ownership on hospital performance, data is collected from all hospitals in Turkey for a six-year period starting from 2001 to 2006. Data were obtained mainly from the Annual Statistical Health Report published by the Ministry of Health. Hospitals are grouped as: (1) public hospitals, (2) education and research hospitals, (3) university hospitals, and (4) private hospitals. Total number of hospitals in each category change each year, thereby the data set included varying number of hospitals for each year. Yearly distribution of total number of hospitals in our data set is as follows: 2001- 477 hospitals; 2002 - 504 hospitals; 2003 - 526 hospitals; 2004 - 566 hospitals; 2005 - 569 hospitals, $2006-608$ hospitals.

\section{ANALYSES AND RESULTS}

Efficiency Measurement System (EMS) was used to evaluate hospital efficiency. Two assumptions of DEA, Constant Return to Scale (CRS) and Variable Return to Scale (VRS), were used. Hospital efficiencies were evaluated in two different ways. First hospitals were evaluated in their own groups, and second, all the hospitals were evaluated in the same data set. Mean, standard deviation, and minimum value of the efficiency scores of the hospitals and the number of efficient hospitals are given in the Table 1 with the assumptions of CRS and VRS. These scores are the average value of the efficiencies in the six years period.

\section{Table I Average Efficiency Measures of Hospitals}

\begin{tabular}{|c|c|c|c|c|c|c|c|c|c|}
\hline & & \multicolumn{4}{|c|}{ CRS } & \multicolumn{4}{|c|}{ VRS } \\
\hline & & $\begin{array}{c}\text { Meara. } \\
\%\end{array}$ & $\begin{array}{l}\text { Std. } \\
\text { Dev. } \\
\%\end{array}$ & $\begin{array}{c}\text { Mina. } \\
\%\end{array}$ & $\begin{array}{l}\text { Nurnbex of } \\
\text { Efficiext } \\
\text { Hospitals }\end{array}$ & $\begin{array}{c}\text { Mean. } \\
\%\end{array}$ & $\begin{array}{l}\text { Std. } \\
\text { Dev. } \\
\%\end{array}$ & $\begin{array}{c}\text { Min. } \\
\%\end{array}$ & $\begin{array}{l}\text { Nuxruber of } \\
\text { Efficiext } \\
\text { Hospitals }\end{array}$ \\
\hline \multirow[t]{2}{*}{ State Hosp. } & State & 70.80 & 18.52 & 23.88 & 52.67 & 77.10 & 18.33 & 24.44 & 89.66 \\
\hline & All & 65.76 & 18.15 & 22.60 & 34.67 & 73.29 & 18.74 & 23.12 & 66.83 \\
\hline Ed.\&R.es. & Ed.\&Res. & 92.68 & 9.79 & 73.91 & 9.17 & 99.08 & 2.11 & 91.47 & 14.16 \\
\hline Hosp. & All & 51.02 & 23.19 & 25.79 & 1.33 & 79.75 & 22.37 & 40.66 & 6 \\
\hline Unuiv. & Univ. & 85.26 & 16.86 & 41.63 & 14.67 & 96.74 & 7.67 & 67.75 & 30.33 \\
\hline Hosp. & All & 32.96 & 13.76 & 13.89 & 0.50 & 73.82 & 22.69 & 28.52 & 13.83 \\
\hline Private & Private & 68.14 & 23.30 & 23.64 & 22.83 & 76.55 & 22.06 & 28.75 & 38.16 \\
\hline Hosp. & All & 54.23 & 25.10 & 16.79 & 24.17 & 67.44 & 2324 & 23.30 & 32.16 \\
\hline
\end{tabular}

Under CRS assumption, university hospitals have the minimum average efficiency score (\%32.96) when all hospital groups are evaluated together. However, when university hospitals are evaluated among themselves, the efficiency score increase to the \%85.26. Similarly the average efficiency score of the education-research hospitals is $\% 92.68$ when isolated from other hospital groups, but it decreases to $\% 51.02$ when all the hospital groups are evaluated together.

The difference between the efficiency scores under the assumptions of CRS and VRS is caused from the differences of the scale efficiency. CRS efficiency scores of the education-research and the university hospitals are significantly less than the corresponding VRS efficiency scores. Thus, scale efficiencies of the education-research and the university hospitals are less than the scale efficiencies of state and private hospitals meaning that the changes in inputs will have a relatively small effect on the outputs.

All hospitals are compared to the hospital(s) which performed in the most efficient scale in the CRS assumption. On the other hand, in VRS assumption any hospital $\mathrm{k}$ is compared to the $\% 100$ technical efficient hospitals (but not necessarily 100\% scale-efficient) (Brown and Pagan, 2006). Therefore, VRS efficiency score is always equal or more than CRS efficiency score. Education \& research and the university hospitals have been found less efficient than the state and private hospitals under the evaluation of scale efficiency. 


\section{Relationship between Hospital Ownership and Efficiency Score}

Statistically differences between the efficiencies of the four groups of hospitals were analyzed by using Kruskal-Wallis and One-Way-Anova analysis. Results are shown in Table 2. According to the findings, hospital efficiencies significantly differentiate by the hospital ownership for all years.

\section{Table II Test Results for the Differences of Hospital Efficiencies by Ownership}

\begin{tabular}{ccc|c|ccc}
\hline \multicolumn{3}{c}{ Kruskal-Wallis } & & \multicolumn{3}{c}{ One-Way-Anova } \\
Year & Ki-kare & d.f. & & Year & F & d.f. \\
\hline 2001 & $40,365^{*}$ & 3 & & 2001 & $19,906^{*}$ & 3 \\
2002 & $38,980^{*}$ & 3 & & 2002 & $18,461^{*}$ & 3 \\
2003 & $36,123^{*}$ & 3 & & 2003 & $16,362^{*}$ & 3 \\
2004 & $33,779^{*}$ & 3 & & 2004 & $16,641^{*}$ & 3 \\
2005 & $30,703^{*}$ & 3 & & 2005 & $13,649^{*}$ & 3 \\
2006 & $47,439^{*}$ & 3 & & 2006 & $33,743^{*}$ & 3 \\
\hline
\end{tabular}

It is reasonable to say that education-research and university hospitals have a completely different structure from the state and private hospitals since they are more oriented towards scientific development and training. State hospitals and private hospitals were also shown to be different in terms of their efficiencies. Our findings in Table 2 are in line with the results of the previous studies. In addition, average efficiencies of state hospitals are higher than the private hospitals for all years.

\section{Super Efficiency Analysis}

All 100\% efficient DMUs will be called efficient in DEA. However it may not be argued that the efficiencies of all efficient DMUs are the same. Ranking among these efficient hospitals can be made by using the super-efficiency score ( $\mathrm{Zhu}, 2003)$. The mathematical structure of the super efficiency analyses is shown in the following (Ramanathan, 2005); for,

$$
\begin{gathered}
\sum_{i=1}^{I} u_{m i} x_{m i}=1 ; \\
\sum_{J=1}^{J} v_{m j} y_{m j}-\sum_{i=1}^{I} u_{m i} x_{m i} \leq 0 ; n=1,2, \ldots ., N ; n \neq m \\
\max \sum_{j=1}^{J} v_{m j} y_{m j} \quad \begin{array}{l}
v_{m j}, u_{m i} \geq 0 ; i=1,2, \ldots \ldots, I ; j=1,2, \ldots \ldots ., J
\end{array}
\end{gathered}
$$

According to the analyses state and private hospitals have been found high efficiency level in the ranking of super efficient hospitals. On the other hand, most of the education-research and university hospitals are in the bottom side of the ranking.

\section{Slack Evaluations}

Inefficiency is caused by non-effective use of the inputs and/or outputs (Cooper et al., 2004). Therefore evaluation of the slack for inputs and outputs is crucial for efficiency improvement. Some variables that controllable with managerial activities, namely the number of specialists, number of medical practitioners, number of beds, number of outpatients and number of discharge, were evaluated based on their average slack values (Table 3).

Slack values show that number of medical practitioners should be decreased for research-education hospitals and university hospitals. On the output side, outpatient number should be increased for all groups of hospitals. Outpatient number seems awfully insufficient for university hospitals. However outpatient number does not show that much slack for education-research hospitals. Managers of university hospitals should investigate the causes of the slack for the number of outpatients. In addition, education \& research hospitals can be a role model for university hospitals in terms of outpatient slack. The slack of discharge number is nearly same for the state and private hospitals thereby the improvement actions for increasing discharge numbers might be similar in these hospitals. 
Table III Average Slacks of Inefficient Hospitals

\begin{tabular}{lccccc}
\hline & \multicolumn{3}{c}{ Input Slacks } & \multicolumn{2}{c}{ Output Slacks } \\
\cline { 2 - 6 } & $\begin{array}{c}\text { Number of } \\
\text { Beds }\end{array}$ & $\begin{array}{c}\text { Number of } \\
\text { Specialists }\end{array}$ & $\begin{array}{c}\text { Number of } \\
\text { Medical } \\
\text { Practitioners }\end{array}$ & $\begin{array}{c}\text { Number of } \\
\text { Outpatients }\end{array}$ & $\begin{array}{c}\text { Number of } \\
\text { Discharge }\end{array}$ \\
\cline { 2 - 6 } $\begin{array}{l}\text { State Hospitals } \\
\begin{array}{l}\text { Education\& } \\
\text { Research }\end{array}\end{array}$ & 0 & 1.6 & 6.6 & 15474.3 & 826.3 \\
$\begin{array}{l}\text { Hospitals } \\
\begin{array}{l}\text { University } \\
\text { Hospitals }\end{array}\end{array}$ & 0.1 & 0.6 & 99.5 & 3278 & 5016.8 \\
$\begin{array}{l}\text { Private } \\
\text { Hospitals }\end{array}$ & 7 & 3.3 & 58.1 & 76184.1 & 5548.6 \\
\hline
\end{tabular}

The evaluation of the slacks is shown that improvement activities should be implemented for all groups of hospitals. According to the findings state and private hospitals have the similar slack values for inputs and outputs while education-research and university hospitals have similar slacks. Hospital managers attend to this case for the planning of improvement activities.

\section{Patterns of Efficiency Changes Over the Period 2001-2006}

Malmquist Productivity Index (MPI) can be used to measure the patterns of efficiency changes on the panel data (Zhu, 2003). Malmquist Productivity Index is defined as follows:

$$
M^{t+1}\left(x^{t+1}, y^{t+1}, x^{t}, y^{t}\right)=\left[\frac{D^{t}\left(x^{t+1}, y^{t+1}\right)}{D^{t}\left(x^{t}, y^{t}\right)} \times \frac{D^{t+1}\left(x^{t+1}, y^{t+1}\right)}{D^{t+1}\left(x^{t}, y^{t}\right)}\right]^{1 / 2}
$$

$\mathrm{D}^{t}$ is a distance function measuring the efficiency of conversion of inputs $\mathrm{x}^{\mathrm{t}}$ to outputs $\mathrm{y}^{\mathrm{t}}$ in period $\mathrm{t}$. As Chiu et al. (2008) noted, we assume that the distance functions are a trans-log form with matching second-order terms, therefore technology is piecewise linear, which allows inefficiencies. MPI can also be considered as a geometric average of the effect of technology change and it can be written as:

$$
M^{t+1}\left(x^{t+1}, y^{t+1}, x^{t}, y^{t}\right)=\left[\frac{D^{t+1}\left(x^{t+1}, y^{t+1}\right)}{D^{t}\left(x^{t}, y^{t}\right)}\right] \times\left[\frac{D^{t}\left(x^{t+1}, y^{t+1}\right)}{D^{t+1}\left(x^{t+1}, y^{t+1}\right)} \times \frac{D^{t}\left(x^{t}, y^{t}\right)}{D^{t+1}\left(x^{t}, y^{t}\right)}\right]^{1 / 2}
$$

Performance change of hospitals in a six-year period is evaluated by using Malmquist Productivity Index. The efficiency changes from 2001-2002, 2002-2003, 2003-2004, 2004-2005 and 2005-2006 are evaluated. If MPI exceeds unity there has been an improvement in productivity between periods T0 and $\mathrm{T} 1$ contrary the measures less than 1 . Only the hospitals which are in data set for all six year period can be analyzed by MPI technique. Therefore many hospitals were eliminated from the data. The remaining 198 state hospitals, 2 education-research hospitals, 26 university hospitals and 24 private hospitals were analyzed. Yearly MPI values of four groups of hospitals are shown in Table 4. The body of the table shows how many hospitals' MPI values exceed $1(>1)$, how many equal $1(=1)$, and how many is below $1(<1)$. It is interesting that state hospitals' MPI measures seriously increase in 2003-2004. Therefore technical efficiencies of state hospitals markedly increased for this period. 
Table IV MPI Values of Hospital Groups $<$

\begin{tabular}{l|c|ccccc}
\hline & & \multicolumn{5}{c}{ Hospital Number } \\
\cline { 3 - 7 } & MPI & $2001-2002$ & $2002-2003$ & $2003-2004$ & $2004-2005$ & $2005-2006$ \\
\hline \multirow{2}{*}{ State Hospitals } & $>1$ & 89 & 86 & 158 & 118 & 105 \\
$(198)$ & $=1$ & 14 & 12 & 9 & 18 & 11 \\
& $<1$ & 95 & 100 & 31 & 62 & 82 \\
\hline Education\& & $>1$ & 1 & 1 & 2 & 0 & 2 \\
Research Hospitals & $=1$ & 0 & 0 & 0 & 1 & 0 \\
$(2)$ & $<1$ & 1 & 1 & 0 & 1 & 0 \\
\hline \multirow{2}{*}{ University Hospitals } & $>1$ & 16 & 15 & 20 & 9 & 16 \\
$(26)$ & $=1$ & 0 & 0 & 0 & 2 & 10 \\
& $<1$ & 10 & 11 & 6 & 7 & 10 \\
\hline \multirow{2}{*}{ Private Hospitals } & $>1$ & 11 & 9 & 6 & 7 & 5 \\
$(24)$ & $=1$ & 6 & 6 & 9 & 10 & 7 \\
\hline
\end{tabular}

Increased efficiency of state hospitals in this period might be correlated with the health care reforms. Turkish Ministry of Health started hospital performance reforms in 2003 (Aydın and Demir, 2006). As can be seen from the average efficiency values in Table 1, the average efficiency of private hospitals clearly decreases in 2004. According to the MPI values in Table 4, there is a serious alteration in the efficiencies of state hospitals and private hospitals in 2003 and 2004. In addition, Wilcoxon and paired sample $t$ tests are applied to evaluate the significant differences between the hospital efficiencies for these years (see in Table 5).

Table V Significant Differences between the Hospital Efficiencies

\begin{tabular}{|c|c|c|c|c|}
\hline \multicolumn{2}{|c|}{ Wilcoxon test } & \multicolumn{3}{|c|}{ Paired-sample $t$ test } \\
\hline Year & Z & Year & $\mathrm{t}$ & d.f. \\
\hline $2001-2002$ & $-1,457$ & $2001-2002$ & $1,923 *$ & 249 \\
\hline $2002-2003$ & $-2,282 * *$ & $2002-2003$ & $1,895^{*}$ & 249 \\
\hline $2003-2004$ & $-4,192 * * *$ & 2003-2004 & $3.871 * * *$ & 249 \\
\hline $2004-2005$ & $-0,188$ & $2004-2005$ & 0,790 & 249 \\
\hline $2005-2006$ & $-0,481$ & $2005-2006$ & 0,959 & 249 \\
\hline
\end{tabular}

According to the findings it can be shown that there is a significant difference between the hospital efficiencies. The degree of significant differences is higher in 2003 and 2004. Consequently it can be argued that health care reforms which have started in 2003 might have an effect on hospital efficiencies (especially for the state and private hospitals).

\section{CONCLUSION}

In this paper, the comparative efficiencies of hospitals have been assessed using Data Envelopment Analysis. Three inputs and ten outputs have been used in the analysis. Changes in efficiencies of the hospitals over the period 2001-2006 have been analyzed using the Malmquist Productivity Index technique. The results obtained confirmed our expectations concerning the impact of health care reforms on the hospital performance by their ownership.

As discussed in the introduction, the theoretical literature contains several, sometimes conflicting, suggestions that efficiency may differ across ownership types of hospitals. We find empirical evidence of differences of efficiencies across the ownership types. It can be said, as an example, that if all the resources of any state hospital put in the place of, say, a private hospital, the degree of efficiency will be changed. This will also be true among other types of hospital ownerships.

We also investigated the impact of health care reforms on the hospital efficiency. Health care reforms have been started in 2003 and these are still going on. According to our results, the most influence of these reforms is shown for the state and private hospitals. Average efficiencies of state hospitals remarkably increased while the average efficiencies of private hospitals decreased in 2003.

Another interesting finding in this research was that, contrary to our expectations, most of the highly 
efficient hospitals were from the small cities (in terms of population). As usual there are more hospitals in the big cities. However this study showed that there is a negative correlation between the city population and the hospital efficiencies.

Finally, too many hospitals were found inefficient. This means there is considerable room for improvement activities. According to our findings, hospital ownership will have a crucial role in these improvement activities. Therefore, hospital managers and policy makers should understand the basic grounds of efficiency under each type of hospital ownerships.

\section{Acknowledgements}

This study is supported by The Scientific and Technological Research Council of Turkey (Project Number 107K168). 


\section{REFERENCES}

Athanassopoulos, A.D., Gounaris, C. and Sissouras, A. (1999), "A descriptive assessment of the production and cost efficiency of general hospitals in Greece", Health Care Management Science, 2(2):97 $-106$.

Aydın, S. and Demir, M. (2006), Performansa Dayalı Ek Ödeme Sistemi: Sağlıkta Performans Yönetimi, Sağlıkta Dönüşüm Serisi - 2, T.C. Sağlık Bakanlığı Yayınları, Ankara.

Bakar, A.H.A., Hakim, I.L., Chong, S.C., and Lin, B. (2010), "Measuring supply chain performance among public hospital laboratories", International Journal of Productivity and Performance Management, 59(1):75-97.

Banker, R., Charnes, A. and Cooper, W. (1984), "Some models for estimating technical and scale inefficiencies in data envelopment analysis", Management Science, 30:1078-92.

Biqrn, E., Hagen, T.P., Iverson, T. and Magnussen, J. (2003), “The effect of activity-based financing on hospital efficiency: a panel data analysis of DEA efficiency scores 1992-2000", Health Care Management Science, 6(2):271-83.

Brown, H.S. and Pagan, J.A. (2006), "Managed Care and the Scale Efficiency of US Hospitals”, International Journal of Health Care Finance and Economics, 6:278-89.

Burgess, J.F. and Wilson, P.W. (1998), "Variation in inefficiency among US hospitals", INFOR, 36 (3):,84-102.

Chang, H.H. 1998. Determinants of hospital efficiency: the case of central government-owned hospitals in Taiwan. Omega, 26(2):307-317.

Chang, H.H., Chang, W.J., Das, S. and Li, S.H. (2004) "Health care regulation and the operating efficiency of hospitals: evidence from Taiwan", Journal of Accounting and Public Policy, 23(6):483-502.

Charnes, A., Cooper, W.W. and Rhodes, E. (1978), "Measuring the efficiency of decision making units", European Journal of Operational Research, 2(6):429-43.

Chiu, Y., Jan, C., Shen, D.B. and Wang, P.C. (2008), "Efficiency and capital adequacy in Taiwan banking: BCC and super-DEA estimation”, The Service Industries Journal, 28(4):479-96.

Chu, H.L., Liu, S.Z., Romeis, J.C. and Yaung, C.L. (2003), "The initial effects of physicians compensation programs in Taiwan hospitals: implications for staff model HMOs", Health Care Management Science, 6(1):17-26.

Cooper, W.W., Seiford, L.M. and Zhu, J. (2004). Handbook on Data Envelopment Analysis, Kluwer Academic Publishers, Boston.

Farrel, M.J. (1957), "The measurement of productive efficiency", Journal of the Royal Statistical Society: Series A, 120(3):253-81.

Ferrari, A. (2006), "Market oriented reforms of health services: a non-parametric analysis", The Service Industries Journal, 26(1):1-13.

Ferrier, G.D. and Valdmanis, V.G. (2004), “Do mergers improve hospital productivity?" Journal of the Operational Research Society, 55(10):1071-80.

Gannon, B. (2005), "Testing for variation in technical efficiency of hospitals in Ireland", The Economic and Social Review, 36(3):273-94.

Grosskopf, S., Margaritis, D. and Valdmanis, V. (2001), "Comparing teaching and non-teaching hospitals: a frontier approach (teaching vs. non-teaching hospitals)", Health Care Management Science, 4 (2):83-90.

Harris, J., Ozgen, H. and Ozcan, Y. (2000), "Do mergers enhance the performance of hospital efficiency?” Journal of the Operational Research Society, 51:801-11.

Helmig, B. and Lapsley, I. (2001), "On the efficiency of public, welfare and private hospitals in Germany over time: a sectoral DEA study”. Health Services Management Research, 14(4):263-74.

Hofmarcher, M.M., Peterson, I. and Riedel, M. (2002), "Measuring hospital efficiency in Austria - a DEA approach", Health Care Management Science, 5(1):7-14. 
Hollingsworth, B., Dawson, P.J. and Maniadakis, N. (1999), "Efficiency measurement of health care: a review of non-parametric methods and applications", Health Care Management Science, 2(3):16172.

Hu, J.L. and Huang, Y.F. (2004), “Technical efficiencies in large hospitals: a managerial perspective”, International Journal of Management, 21(4):506-13.

Junoy, P.J. (2000), "Partitioning input cost efficiency into its allocative and technical components: an empirical DEA application to hospitals", Socio-Economic Planning Science, 34:199-218.

Kirigia, J.M., Emrouznejad, A., Vaz, R.G., Bastiene, H. and Padayachy, J. (2008), “A comparative assessment of performance and productivity of health centres in Seychelles", International Journal of Productivity and Performance Management, 57(1):72-92.

Kumar, S. and Gulati R. (2008), "Evaluation of technical efficiency and ranking of public sector banks in India", International Journal of Productivity and Performance Management, 57(7):540-68.

Kumar, S. (2010), "Specialty hospitals emulating focused factories", International Journal of Health Care Quality Assurance, 23(1):94-109.

Linna, M., Hakkinen, U. and Magnussen, J. (2006), "Comparing hospital cost efficiency between Norway and Finland", Health Policy, 77:268-78.

Liu, H.H., Chen, Y.T. and Pai, L.Y. (2007), "The Influence of Merger and Acquisition Activities on Corporate Performance in the Taiwanese Telecommunications Industry", The Service Industries Journal, 27(8): 1041-51.

Lynch, J.R. and Ozcan, Y.A. (1994), "Hospital closure: an efficiency analysis", Hospital \& Health Services Administration, 39(2):205-20.

Min, H., Min, H., Joo, S.J. and Kim, J. (2009), "Evaluating the financial performances of Korean luxury hotels using data envelopment analysis", The Service Industries Journal, 29(6):835-45.

Nayar, P. and Ozcan, Y.A. (2008), "Data envelopment analysis comparison of hospital efficiency and quality”, Journal of Medical Systems, 32:193-99.

Nyhan, R.C. and Peter, L.C. (2000), "Comparative performance assessment in managed care: data envelopment analysis for health care manager", Management Care Quarterly, 8(1):18-27.

O'neill, L. and Dexter, F. (2004), "Market capture of inpatient preoperative services using DEA", Health Care Management Science, 7(2):263-73.

Ramanathan, R. (2005) "Operations assessment of hospitals in the Sultanate of Oman", International Journal of Operations \& Production Management, 25(1):39-54.

Sorkis, J. and Talloru, S. (2002), "Efficiency measurement of hospitals: issues and extensions", International Journal of Operation \& Production Management, 22(3):306-13.

Watcharasriroj, B. and Tang, J.C.S. (2004), "The effects of size and information technology on hospital efficiency”, The Journal of High Technology Management Research, 15:1-16.

White, K.R. and Ozcan, Y.A. (1996), "Church ownership and hospital efficiency", Hospital \& Health Services Administration, 41(3):297-310.

Zhu, J. (2003). Quantitative Models for Performance Evaluation and Benchmarking, Springer's International Series, New York. 